\title{
Interpolation of Multi-LPV Control Systems Based on Youla-Kucera Parameterization *
}

\author{
Hussam Atoui $^{\text {a,b }}$, Olivier Sename ${ }^{\text {a }}$, Vicente Milanes ${ }^{\mathrm{b}}$, John J. Martinez ${ }^{\mathrm{a}}$ \\ ${ }^{\mathrm{a}}$ Univ. Grenoble Alpes, CNRS, Grenoble INP, GIPSA-lab, 38000 Grenoble, France \\ ${ }^{\mathrm{b}}$ Research Department, Renault SAS, 1 Avenue de Golf, 78280 Guyancourt, France
}

\begin{abstract}
This paper presents a methodology which considers an interpolation between Linear Parameter-Varying (LPV) controllers that were designed separately for different objectives. The quadratic stability of the closed-loop system is proved under arbitrary interpolation in terms of a set of Linear Matrix Inequalities (LMIs). The interpolation strategy is based on Youla-Kucera (YK) parameterization. The proposed method can help multi-variable and multi-objective systems, to achieve high performances at different critical situations regardless of the interpolation rate.
\end{abstract}

Key words: Linear parameter-varying system, Youla parameterization, Gain-scheduling, Linear matrix inequality

\section{Introduction And Motivation}

The interpolation of several controllers to meet multiobjectives criteria is an important topic in theory and practice. A method has been investigated in the literature to achieve a multi-objective switching Linear TimeInvariant (LTI) controller based on Youla-Kucera (YK) parameterization [5]. It is structured by mapping a set of linear stabilizing controllers onto a $Q$-based controller. The interest is to parameterize a set of linear stabilizing controllers $K(Q)$ for an LTI system, where each one is parameterized by its corresponding LTI-YK parameter $Q$ [14]. The LTI-YK parameter $Q$ is designed using the doubly coprime factorisation [15]. Using such parameterization for controller interpolation: 1) It allows stable gain-scheduling between unstable controllers [13]; 2) Interpolated controllers can be designed and tuned separately using different techniques $\left(\mathcal{H}_{\infty}\right.$, LQR, PID) [14]; 3) It facilitates adding new parts to an existing system online as Plug\&Play control theory [12]; and 4) The closed-loop stability is guaranteed under arbitrary interpolating signals between different stabilizing controllers [5] without requiring a single Lyapunov function.

\footnotetext{
^ Institute of Engineering Univ. Grenoble Alpes

Email addresses: hussam.atoui@renault.com (Hussam Atoui), olivier.sename@grenoble-inp.fr (Olivier Sename), vicente.milanes@renault.com (Vicente Milanes), john-jairo.martinez-molina@grenoble-inp.fr (John J. Martinez).
}

The YK parameterization has been successfully applied to several domains such as: 1) Noise/vibration control [7]; 2) Interpolation between robust and high performance controllers [4]; and 3) Control systems subjected to convex constraints [3]. A significant literature review of YK work including its recent applications can be found in [10]. In addition, YK parameterisation is used in a rising topic which is autonomous vehicles [11],[9]. [9] uses an LTI-YK control structure to interpolate between fast and slow LTI controllers to optimize a lane change of an autonomous vehicle. However, the study is done on an LTI lateral system with a fixed longitudinal speed of the vehicle.

On the other hand, the control of multi-variable or Linear Parameter-Varying (LPV) systems is studied due to its interest to handle system complexity (as nonlinearities) as well as to bring some adaptive control schemes thanks to the self-scheduling of the controller, see for instance [6] and references therein. Nonetheless, it is today admitted that designing a single LPV controller for various objectives may be conservative [8]. However, while many YK interpolation studies have been concerned with LTI systems, few works have integrated YK concepts to LPV systems. [1] introduces an LPV model which varies with respect to time-varying parameters. It proposes a YK-based gain-scheduling between LTI controllers designed separately at different operating points. Closed-loop quadratic stability and performance are guaranteed at intermediate interpolation points of 
a convex domain. The parameterized controller is then compared to an LPV controller designed using the polytopic approach. A different concept is proposed in [16] to design an LPV controller based on Youla parameterisation. It extends the coprime factorization of LTI systems to LPV systems and it is proved that any quadratically stabilizing LPV controller can be parameterized, based on Youla concept, providing the closed-loop quadratic stability. Notice that the concept is proved only for observer-based LPV state-feedback control. In [2], a fixed pole assignment application of LPV system is introduced using an LPV Q-based method. Its concept aims to preserve the closed-loop poles at the same location by interpolating between different controllers regardless the variations in the LPV system. Therefore, a unique performance is obtained along the variation of the parameters while keeping stability.

Nevertheless, none of the mentioned works did concern multi-objective controllers, i.e. achieving different performances in the full parameter-varying region. The objective and contribution of this paper is to develop an LPV-YK dynamic output feedback control structure which can interpolate between several LPV controllers, that have been designed to achieve different objectives. Such interpolation scheme aims to improve the closed-loop performance which could achieve both multi-variable and multi-objective requirements.

This paper develops the work in [16] by proposing a dynamic output-feedback LPV controller (to be parameterized), instead of observer-based state feedback LPV controller, which extends the stability conditions. Moreover, the proposed method extends the work in [1] by adding an exogenous signal (physical-based or parameter-dependent signal) to the model parameters to interpolate between two LPV controllers. This means that new dimensions can be added to the parameter region to incorporate any ad hoc physically-based interpolation. Notice that the addition of such interpolating signal doesn't add any conservatism to the standard LPV problem conservatism. Contrarily, it improves the overall closed-loop performance by choosing the best LPV controller (designed separately) at different situations. As a result, an interpolation scheme is drawn between two LPV controllers based on YK parameterization which guarantees the closed-loop quadratic stability under arbitrary interpolating signal.

\section{Problem Statement}

\subsection{LPV Plant and Controllers Description}

Consider a Multi-Input-Multi-Output (MIMO) LPV system $G(\rho)$ with $m$ inputs and $p$ outputs and $n_{z}$ con- trolled outputs:

$G(\rho)\left\{\begin{array}{l}\dot{x}(t)=A(\rho(t)) x(t)+B_{1}(\rho(t)) w(t)+B_{2} u(t) \\ z(t)=C_{1}(\rho(t)) x(t)+D_{11}(\rho(t)) w(t)+D_{12} u(t) \\ y(t)=C_{2} x(t)+D_{21} w(t)\end{array}\right.$

where $x(t) \in \mathbb{R}^{n_{x}}, y(t) \in \mathbb{R}^{p}, u(t) \in \mathbb{R}^{m}, z(t) \in \mathbb{R}^{n_{z}}$ are the state, output, input, controlled output vectors respectively. $w(t)=\left[\begin{array}{lll}r & n & d\end{array}\right]^{T} \in \mathbb{R}^{n_{w}}$ contains the exogenous inputs of the tracking reference $r$, noise $n$ and input disturbance $d . \rho(t):=\rho \in \mathbb{R}^{n_{p}}$ is a vector of $n_{p}$ known time-varying parameters.

Here, $\rho$ belongs to a convex polytopic region $\mathcal{P}$ defined by the parameters extremums $[\rho, \bar{\rho}]$ as $\mathcal{P}:=$ $\mathcal{C}_{\mathcal{O}}\left\{\nu_{1}, \ldots, \nu_{2^{n_{p}}}\right\}$, where $\nu_{i}$ represent the vertices of $\mathcal{P}$ $\forall i \in \mathbb{I}\left[1,2^{n_{p}}\right] . \rho$ is then scheduled as: $\rho=\sum_{i=1}^{2^{n_{p}}} \alpha_{i} \nu_{i}$, where $\sum_{i=1}^{2^{n} p} \alpha_{i}=1, \alpha_{i} \geq 0 \forall i$. Therefore, the system representation at any operating point $\rho \in \mathcal{P}$ is given as a convex combination of the state-space realizations of the LTI systems given at the vertices $\nu_{i}$ :

$$
\left[\begin{array}{c|cc}
A(\rho) & B_{1}(\rho) & B_{2} \\
\hline C_{1}(\rho) & D_{11}(\rho) & D_{12} \\
C_{2} & D_{21} & 0
\end{array}\right]=\sum_{i=1}^{2^{n_{p}}} \alpha_{i}(\rho)\left[\begin{array}{c|cc}
A_{i} & B_{1, i} & B_{2} \\
\hline C_{1, i} & D_{11, i} & D_{12} \\
C_{2} & D_{21} & 0
\end{array}\right]
$$

Let $\mathcal{K}$ be a finite set of quadratically stabilizing LPV controllers $K^{(j)}(\rho)$ of $G(\rho)$ that were designed to achieve different objectives and performances. Thus, $\forall j \in \mathbb{I}\left[0, n_{c}\right]$ :

$$
K^{(j)}(\rho):\left[\begin{array}{c|c}
A_{k}^{(j)}(\rho) & B_{k}^{(j)}(\rho) \\
\hline C_{k}^{(j)}(\rho) & D_{k}^{(j)}(\rho)
\end{array}\right]=\sum_{i=1}^{2^{n_{p}}} \alpha_{i}(\rho)\left[\begin{array}{c|c}
A_{k, i}^{(j)} & B_{k, i}^{(j)} \\
\hline C_{k, i}^{(j)} & D_{k, i}^{(j)}
\end{array}\right]
$$

where $A_{k}^{(j)}(\rho) \in \mathbb{R}^{n_{k}^{(j)} \times n_{k}^{(j)}}, B_{k}^{(j)}(\rho) \in \mathbb{R}^{n_{k}^{(j)} \times m_{k}}$, $C_{k}^{(j)}(\rho) \in \mathbb{R}^{p_{k} \times n_{k}^{(j)}}$ and $D_{k}^{(j)}(\rho) \in \mathbb{R}^{p_{k} \times m_{k}}$.

Notation 2.1 For the rest of the paper, the subscript $i$ of a matrix (resp. system) denotes the LTI matrix (resp. system) at its corresponding vertex $\nu_{i}$ of the polytope $\mathcal{P}$. The superscript $(j)$ denotes the $j^{\text {th }}$ controller in the set of already designed LPV controllers $\mathcal{K}$.

\subsection{Coprime Factorization}

Using the YK parameterisation concept, the plant model $G(\rho)$ and the controllers $K^{(j)}(\rho)$ given at each vertex $\nu_{i}$ of $\mathcal{P}$ (namely $G_{i}$ and $K_{i}^{(j)}$ ), can be factorized using the 


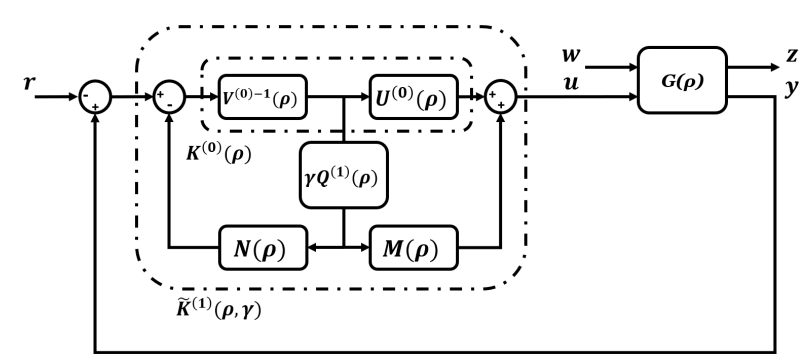

Fig. 1. YK control structure

doubly coprime factorisation as $\forall i, j$ :

$$
\begin{gathered}
G_{i}=N_{i} M_{i}^{-1}=\tilde{M}_{i}^{-1} \tilde{N}_{i} \quad \forall i \\
K_{i}^{(j)}=U_{i}^{(j)} V_{i}^{(j)-1}=\tilde{V}_{i}^{(j)-1} \tilde{U}_{i}^{(j)} \quad \forall i, \forall j
\end{gathered}
$$

Thus $\forall i, \forall j$, the coprime factors are computed such that $M_{i}, N_{i}, \tilde{M}_{i}, \tilde{N}_{i}, U_{i}^{(j)}, V_{i}^{(j)}, \tilde{U}_{i}^{(j)}, \tilde{V}_{i}^{(j)} \in \mathcal{R} \mathcal{H}_{\infty}$ (proper, stable and rational) [14] and satisfying the following $B e$ zout Identity:

$$
\left[\begin{array}{cc}
\tilde{V}_{i}^{(j)} & -\tilde{U}_{i}^{(j)} \\
-\tilde{N}_{i} & \tilde{M}_{i}
\end{array}\right]\left[\begin{array}{cc}
M_{i} & U_{i}^{(j)} \\
N_{i} & V_{i}^{(j)}
\end{array}\right]=\left[\begin{array}{cc}
M_{i} & U_{i}^{(j)} \\
N_{i} & V_{i}^{(j)}
\end{array}\right]\left[\begin{array}{cc}
\tilde{V}_{i}^{(j)} & -\tilde{U}_{i}^{(j)} \\
-\tilde{N}_{i} & \tilde{M}_{i}
\end{array}\right]=\left[\begin{array}{ll}
I & 0 \\
0 & I
\end{array}\right]
$$

At each vertex $\nu_{i}$, all the factorized LTI controllers $K_{i}^{(j)}$ stabilize $G_{i}$ (proof in [14]). To perform a stable coprime factorisation, polytopic LPV state-feedback controllers $F_{g}(\rho)$ and $F_{k}^{(j)}(\rho)$ are designed to quadratically stabilize $G(\rho)$ and $K^{(j)}(\rho)$ respectively $\forall j$. This ensures the structural stability of the closed-loops $\left(A_{i}+B_{2} F_{g, i}\right)$ and $\left(A_{k, i}^{(j)}+B_{k, i}^{(j)} F_{k, i}^{(j)}\right)$ which are Hurwitz $\forall i, j$. The coprime factors are computed at each vertex $\nu_{i}$ using the statespace representations written in (6)-(7).

$$
\left[\begin{array}{cc}
M_{i} & U_{i}^{(j)} \\
N_{i} & V_{i}^{(j)}
\end{array}\right]:\left[\begin{array}{cc|cc}
A_{i}+B_{2} F_{g, i} & 0 & B_{2} & 0 \\
0 & A_{k, i}^{(j)}+B_{k, i}^{(j)} F_{k, i}^{(j)} & 0 & B_{k, i}^{(j)} \\
\hline F_{g, i} & C_{k, i}^{(j)}+D_{k, i}^{(j)} F_{k, i}^{(j)} & I & D_{k, i}^{(j)} \\
C_{2} & F_{k, i}^{(j)} & 0 & I
\end{array}\right]
$$

$$
\left[\begin{array}{cc}
\tilde{V}_{i}^{(j)} & -\tilde{U}_{i}^{(j)} \\
-\tilde{N}_{i} & \tilde{M}_{i}
\end{array}\right]:\left[\begin{array}{cc|cc}
A_{i}+B_{2} D_{k, i}^{(j)} C_{2} & B_{2} C_{k, i}^{(j)} & -B_{2} & B_{2} D_{k, i}^{(j)} \\
B_{k, i}^{(j)} C_{2} & A_{k, i}^{(j)} & 0 & B_{k, i}^{(j)} \\
\hline F_{g, i}-D_{k, i}^{(j)} C_{2} & -C_{k, i}^{(j)} & I & -D_{k, i}^{(j)} \\
C_{2} & -F_{k, i}^{(j)} & 0 & I
\end{array}\right]
$$

\subsection{The LPV-YK Control Structure}

Fig. 1 shows the proposed structure of the dynamic interpolation between two dynamic output-feedback LPV controllers $K^{(0)}(\rho)$ and $K^{(1)}(\rho) \in \mathcal{K}$. Notice that, as explained above, $N(\rho), M(\rho), U^{(0)}(\rho), V^{(0)-1}(\rho)$ and $Q^{(1)}(\rho, \gamma)$ are computed from the convex combination of their corresponding LTI systems at the vertices of $\mathcal{P}$. $K^{(0)}(\rho)$ is chosen to be the nominal controller, where it can be designed to achieve high robustness. The remaining controllers (such as $K^{(1)}(\rho)$ ) can be designed to deal with higher performances or critical situations. Then, $\forall i, K_{i}^{(1)}$ is written in terms of its corresponding YK parameter $Q_{i}^{(1)}$ represented as:

$$
Q_{i}^{(1)}:\left[\begin{array}{c|c}
A_{q, i}^{(1)} & B_{q, i}^{(1)} \\
\hline C_{q, i}^{(1)} & D_{q, i}^{(1)}
\end{array}\right]
$$

Following Fig. 1, in this work, an external signal $\gamma(t)$ multiplying the output of the designed $Q_{i}^{(1)}$, i.e. its output matrices are written as $C_{q, i}^{(1)}(\gamma)=\gamma(t) C_{q, i}^{(1)}$ and $D_{q, i}^{(1)}(\gamma)=\gamma(t) D_{q, i}^{(1)}$ obtaining $Q_{i}^{(1)}(\gamma)=\gamma Q_{i}^{(1)}$ (see Fig. 1). $\gamma$ could be any continuous or discontinuous switching signal which interpolates between $K^{(0)}(\rho)$ and $K^{(1)}(\rho)$. This complete interpolated LPV controller is referred to as the parameterized controller $\tilde{K}^{(1)}(\rho, \gamma)$. The statespace representation of $Q^{(1)}(\rho, \gamma)$ is represented as:

$$
Q^{(1)}(\rho, \gamma):\left[\begin{array}{c|c}
A_{q}^{(1)}(\rho) & B_{q}^{(1)}(\rho) \\
\hline C_{q}^{(1)}(\rho, \gamma) & D_{q}^{(1)}(\rho, \gamma)
\end{array}\right]
$$

where $A_{q}^{(1)} \in \mathbb{R}^{n_{q}^{(1)} \times n_{q}^{(1)}}$. The interpolated LPV-YK controller $\tilde{K}^{(1)}(\rho, \gamma)$ is computed as:

$$
\begin{aligned}
\tilde{K}^{(1)}(\rho, \gamma) & =\left(U^{(0)}(\rho)+M(\rho) Q^{(1)}(\rho, \gamma)\right)\left(V^{(0)}(\rho)+N(\rho) Q^{(1)}(\rho, \gamma)\right)^{-1} \\
& =\left(\tilde{V}^{(0)}(\rho)+Q^{(1)}(\rho, \gamma) \tilde{N}(\rho)\right)^{-1}\left(\tilde{U}^{(0)}(\rho)+Q^{(1)}(\rho, \gamma) \tilde{M}(\rho)\right)
\end{aligned}
$$

Its state space matrices are written as:

$$
\begin{gathered}
\tilde{A}_{k}^{(1)}(\rho, \gamma)=\left[\begin{array}{ccc}
A(\rho)+B_{2} F_{g}(\rho)-B_{2} D_{q}^{(1)}(\rho, \gamma) C_{2} & -B_{2} D_{q}^{(1)}(\rho, \gamma) F_{k}^{(0)}(\rho) & B_{2} C_{q}^{(1)}(\rho, \gamma) \\
-B_{k}^{(0)}(\rho) C_{2} & A_{k}^{(0)}(\rho) & 0 \\
-B_{q}^{(1)}(\rho) C_{2} & -B_{q}^{(1)}(\rho) F_{k}^{(0)}(\rho) & A_{q}^{(1)}(\rho)
\end{array}\right] \\
\tilde{B}_{k}^{(1)}(\rho, \gamma)=\left[B_{2} D_{q}^{(1)}(\rho, \gamma) B_{k}^{(0)}(\rho) B_{q}^{(1)}(\rho)\right]^{T} \\
\tilde{C}_{k}^{(1)}(\rho, \gamma)=\left[F_{g}(\rho)-\left(D_{k}^{(0)}(\rho)+D_{q}^{(1)}(\rho, \gamma)\right) C_{2} C_{k}^{(0)}(\rho)-D_{q}^{(1)}(\rho, \gamma) F_{k}^{(0)}(\rho) C_{q}^{(1)}(\rho, \gamma)\right] \\
\tilde{D}_{k}^{(1)}(\rho, \gamma)=D_{k}^{(0)}(\rho)+D_{q}^{(1)}(\rho, \gamma)
\end{gathered}
$$

The next step proposes a gain-scheduling/interpolation between the designed LPV controllers to achieve different performances depending on the situations. Knowing that $Q^{(1)}(\rho, \gamma)$ is quadratically stable by construction (see later in the proof of Theorem 3.1), it is worth mentioning that:

- for $\gamma=0, \tilde{K}^{(1)}(\rho, \gamma=0)=K^{(0)}(\rho)$

- for $\gamma=1, \tilde{K}^{(1)}(\rho, \gamma=1)=K^{(1)}(\rho)$ 


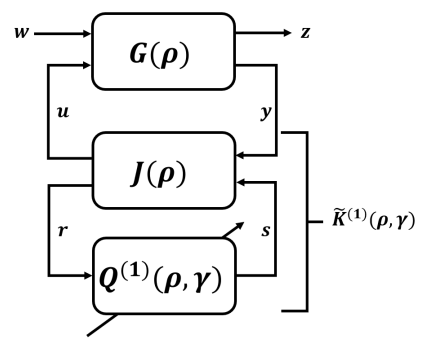

Fig. 2. YK general configuration

\section{Main Results}

Based on the statements on LPV concepts and YK parameterization, a quadratically stable interpolation procedure between two LPV controllers is formulated. A non-minimal state-space realization of each LPV controller is computed based on Linear Matrix Inequality (LMI) optimization problem [5]. A gain-scheduling signal $\gamma$ is included to interpolate between both controllers.

Theorem 3.1 Consider an LPV plant $G(\rho)$ (2), and given a set of LPV controllers $\mathcal{K}$ (3) that quadratically stabilizes $G(\rho)$. Let us choose any two controllers $K^{(0)}(\rho)$ and $K^{(1)}(\rho) \in \mathcal{K}$, where $K^{(0)}(\rho)$ represents the nominal controller. Then, the YK-parameterized LPV controller $\tilde{K}^{(1)}(\rho, \gamma)(11)$ (see Fig. 1) stabilizes $G(\rho)$ for any continuous/discontinuous signal $\gamma \in[0,1]$, if there exist symmetric, positive definite matrices $X_{g} \in \mathbb{R}^{n_{x} \times n_{x}}$, $X_{k} \in \mathbb{R}^{n_{k}^{(0)} \times n_{k}^{(0)}}$, and matrices $W_{i}$ and $V_{i}$ such that:

$$
\begin{gathered}
A_{i} X_{g}+X_{g} A_{i}^{T}+B_{2} W_{i}+W_{i}^{T} B_{2}^{T}<0 \\
A_{k, i}^{(0)} X_{k}+X_{k} A_{k, i}^{(0) T}+B_{k, i}^{(0)} V_{i}+V_{i}^{T} B_{k, i}^{(0) T}<0
\end{gathered}
$$

for all $i=1, \ldots, 2^{n_{p}}$.

PROOF. According to YK parameterisation concept, $\forall \rho \in \mathcal{P}$, the LPV parameterized controller $\tilde{K}^{(1)}(\rho, \gamma)$ can be formulated as a Linear Fractional Transformation (LFT) system [14], i.e. $\tilde{K}^{(1)}(\rho, \gamma)=\mathcal{F}_{l}\left(J(\rho), Q^{(1)}(\rho, \gamma)\right)$ (see Fig. 2). Where, $J(\rho)$ and $Q^{(1)}(\rho, \gamma)$ are written as a convex combination of their corresponding LTI systems (14)-(15) at the vertices $\nu_{i}$ of $\mathcal{P}$.

$$
J_{i}=\left[\begin{array}{cc|cc}
A_{i}+B_{2} F_{g, i} & 0 & 0 & B_{2} \\
-B_{k, i}^{(0)} C_{2} & A_{k, i}^{(0)} & B_{k, i}^{(0)} & 0 \\
\hline F_{g, i}-D_{k, i}^{(0)} C_{2} & C_{k, i}^{(0)} & D_{k, i}^{(0)} & I \\
-C_{2} & -F_{k, i}^{(0)} & I & 0
\end{array}\right]
$$

$$
\begin{aligned}
& Q_{i}^{(1)}(\gamma)= \\
& {\left[\begin{array}{ccc|c}
A_{i}+B_{2} D_{k, i}^{(1)} C_{2} & B_{2} C_{k, i}^{(1)} & B_{2}\left[D_{k, i}^{(1)}-D_{k, i}^{(0)}\right] F_{k, i}^{(0)}-B_{2} C_{k, i}^{(0)} & B_{2}\left[D_{k, i}^{(1)}-D_{k, i}^{(0)}\right] \\
B_{k, i}^{(1)} C_{2} & A_{k, i}^{(1)} & B_{k, i}^{(1)} F_{k, i}^{(0)} & B_{k, i}^{(1)} \\
\hdashline 0 & 0 & A_{k, i}^{(0)}+B_{k, i}^{(0)} F_{k, i}^{(0)} & B_{k, i}^{(0)} \\
\hline \gamma\left[D_{k, i}^{(1)} C_{2}-F_{g, i}\right] & \gamma C_{k, i}^{(1)} & \gamma\left[\left(D_{k, i}^{(1)}-D_{k, i}^{(0)}\right) F_{k, i}^{(0)}-C_{k, i}^{(0)}\right] & \gamma\left[D_{k, i}^{(1)}-D_{k, i}^{(0)}\right]
\end{array}\right]}
\end{aligned}
$$

At each vertex $\nu_{i}$, the closed-loop system $C L_{i}(\gamma)$ is derived from the LFT interconnection between $G_{i}$ and $\tilde{K}_{i}^{(1)}(\gamma)$. The closed-loop state matrix $A_{c l}(\rho, \gamma)=$ $\sum_{i=1}^{2^{n} p} \alpha_{i}(\rho) A_{c l, i}(\gamma)$ is quadratically stable if there exist a symmetric, positive definite, constant matrix $X_{c l}$ such that:

$X_{c l} A_{c l}(\rho, \gamma)+A_{c l}^{T}(\rho, \gamma) X_{c l}<0 \forall \gamma$
Now, let $T=\left[\begin{array}{cccc}I & 0 & 0 & 0 \\ 0 & 0 & 0 & I \\ I & -I & 0 & 0 \\ 0 & 0 & I & 0\end{array}\right]$ be a state transformation matrix which is applied to $C L_{i}(\gamma)$ without changing its input-output nature, then

$$
\begin{aligned}
& \bar{A}_{c l, i}(\gamma)=T A_{c l, i}(\gamma) T^{-1}= \\
& {\left[\begin{array}{cccc}
A_{i}+B_{2} F_{g, i} & B_{2} C_{q, i}^{(1)} & -B_{2}\left(F_{g, i}-\left(D_{k, i}^{(0)}+D_{q, i}^{(1)}(\gamma)\right) C_{2}\right) & B_{2}\left(C_{k, i}^{(0)}-D_{q, i}^{(1)} F_{k, i}^{(0)}\right) \\
0 & A_{q, i}^{(1)} & B_{q, i}^{(1)} C_{2} & -B_{q, i}^{(1)} F_{k, i}^{(0)} \\
\hdashline 0 & 0 & A_{i}+B_{2} D_{k, i}^{(0)} C_{2} & B_{2} C_{k, i}^{(0)} \\
0 & 0 & B_{k, i}^{(0)} C_{2} & A_{k, i}^{(0)}
\end{array}\right]}
\end{aligned}
$$

Due to the block-triangular form of $\bar{A}_{c l}(\rho, \gamma)(17),(16)$ is satisfied if the following equations hold (check Lemma 2 in $[16])$ :

$$
\begin{aligned}
\sum_{i=1}^{2^{n_{p}}} \alpha_{i}(\rho) & \left(Y_{g}\left(A_{i}+B_{2} F_{g, i}\right)+\left(A_{i}+B_{2} F_{g, i}\right)^{T} Y_{g}\right)<0 \\
& \sum_{i=1}^{2^{n_{p}}} \alpha_{i}(\rho)\left(Y_{q} A_{q, i}^{(1)}+A_{q, i}^{(1) T} Y_{q}\right)<0 \\
& \sum_{i=1}^{2^{n_{p}}} \alpha_{i}(\rho)\left(Y_{0} \mathcal{A}_{i}^{(0)}+\mathcal{A}_{i}^{(0) T} Y_{0}\right)<0
\end{aligned}
$$

where $Y_{g} \in \mathbb{R}^{n_{x} \times n_{x}}, Y_{q} \in \mathbb{R}^{n_{q}^{(1)} \times n_{q}^{(1)}}$ and $Y_{0} \in$ $\mathbb{R}^{\left(n_{x}+n_{k}^{(0)}\right) \times\left(n_{x}+n_{k}^{(0)}\right)}$ are symmetric, positive definite, parameter-invariant matrices, with $X_{c l}=T^{T}$ $\operatorname{diag}\left(Y_{g}, Y_{q}, Y_{0}\right) T$, and

$$
\mathcal{A}_{i}^{(0)}=\left[\begin{array}{cc}
A_{i}+B_{2} D_{k, i}^{(0)} C_{2} & B_{2} C_{k, i}^{(0)} \\
B_{k, i}^{(0)} C_{2} & A_{k, i}^{(0)}
\end{array}\right]
$$

Inequality (18) can be reformulated by $Y_{g}=X_{g}^{-1}$ which 
leads to (12) when choosing $W(\rho)=\sum_{i=1}^{2^{n} p} \alpha_{i}(\rho) F_{g, i} X_{g}$. Regarding (15), the state matrix of the LPV-YK parameter $A_{q}^{(1)}(\rho)=\sum_{i=1}^{2^{n_{p}}} \alpha_{i}(\rho) A_{q, i}^{(1)}(\rho)$ is quadratically stable if there exist symmetric, positive definite, constant matrices $P_{1} \in \mathbb{R}^{\left(n_{x}+n_{k}^{(1)}\right) \times\left(n_{x}+n_{k}^{(1)}\right)}$ and $P_{2} \in \mathbb{R}^{n_{k}^{(0)} \times n_{k}^{(0)}}$ such that:

$$
\sum_{i=1}^{2^{n_{p}}} \alpha_{i}(\rho)\left(P_{1} \mathcal{A}_{i}^{(1)}+\mathcal{A}_{i}^{(1) T} P_{1}\right)<0
$$

$\sum_{i=1}^{2^{n_{p}}} \alpha_{i}(\rho)\left(P_{2}\left(A_{k, i}^{(0)}+B_{k, i}^{(0)} F_{k, i}^{(0)}\right)+\left(A_{k, i}^{(0)}+B_{k, i}^{(0)} F_{k, i}^{(0)}\right)^{T} P_{2}\right)<0$

being,

$$
\mathcal{A}_{i}^{(1)}=\left[\begin{array}{cc}
A_{i}+B_{2} D_{k, i}^{(1)} C_{2} & B_{2} C_{k, i}^{(1)} \\
B_{k, i}^{(1)} C_{2} & A_{k, i}^{(1)}
\end{array}\right]
$$

The condition in $(22)$ is verified given that $K^{(1)}(\rho)$ quadratically stabilizes $G(\rho)$. Moreover, the inequality (23) satisfies (13) by choosing $P_{2}=X_{k}^{-1}$ and $V(\rho)=\sum_{i=1}^{2^{n} p} \alpha_{i}(\rho) F_{k, i}^{(0)} X_{k}$. Thus, $A_{q}^{(1)}(\rho)$ is quadratically stable and (19) is verified. Finally, (20) is fulfilled given that $K^{(0)}(\rho)$ quadratically stabilizes $G(\rho)$.

After verifying the conditions of the theorem, it is worth mentioning that they depend only on the nominal LPV controller $K^{(0)}(\rho)$ and not on the other designed LPV controllers given the considered YK parameterization.

The following brief example presents the objective of the proposed approach in improving the closed-loop performance. Given two LPV controllers that are designed separately achieving distinct performance specifications: 1) $K^{(0)}(\rho)$ for noise rejection with slow tracking capabilities; and 2) $K^{(1)}(\rho)$ for fast tracking capabilities but without respecting the robust margin. Let us construct a YK-based interpolation scheme $\widetilde{K}^{(1)}(\rho, \gamma)$ as shown in Fig. 1. As a result, multiple closed-loop performances are obtained thanks to the variation of the interpolating signal $\gamma(t)$. At an instant $t=t_{k}$ :

(1) if $\gamma\left(t_{k}\right)=0, \tilde{K}^{(1)}\left(\rho, \gamma\left(t_{k}\right)=0\right) \equiv K^{(0)}(\rho)$, the closed-loop performance achieves only noise rejection

(2) if $\gamma\left(t_{k}\right)=1, \tilde{K}^{(1)}\left(\rho, \gamma\left(t_{k}\right)=1\right) \equiv K^{(1)}(\rho)$, the closed-loop performance include only tracking capabilities

(3) if $0<\gamma\left(t_{k}\right)<1, \tilde{K}^{(1)}\left(\rho, \gamma\left(t_{k}\right)\right)$ interpolates between both controllers to obtain a percentage of noise rejection performance and a percentage of tracking capabilities.
An example is shown in [9] on how to choose $\gamma$ according to the required control objectives.

\section{Conclusion}

This work has proposed a new LPV YK-based method to design an interpolation between two dynamic outputfeedback LPV controllers. An external signal is introduced to the LPV-YK parameter, which can be used to incorporate any ad-hoc physically-based interpolation. As a result, a YK-based interpolation scheme is drawn between two LPV controllers while guaranteeing the closed-loop quadratic stability under arbitrary interpolating signal. This approach improves the performance for LPV complex systems that need to deal with various objectives and situations. As a future work, the LPV-YK control scheme will be extended to more than two controllers. In addition, application of the presented results and possible extension for discrete-time systems will be explored.

\section{References}

[1] F. Bianchi and R.S. Peña. Interpolation for gain-scheduled control with guarantees. Automatica, 47(1):239-243, 2011.

[2] F. Blanchini, D. Casagrande, S. Miani, and U. Viaro. Stable lpv realization of parametric transfer functions and its application to gain-scheduling control design. IEEE Transactions on Automatic Control, 55(10):2271-2281, Oct 2010.

[3] O. Cifdaloz, A. A. Rodriguez, and J. M. Anderies. Control of distributed parameter systems subject to convex constraints: Applications to irrigation systems and hypersonic vehicles. In 200847 th IEEE Conference on Decision and Control, pages 865-870, 2008.

[4] J. Hespanha and A. S. Morse. Towards the high performance control of uncertain processes via supervision. In In Proc. of the 30th Annual Conf. on Information Sciences and Syst, pages 405-410, 1996.

[5] J. Hespanha and A. S. Morse. Switching between stabilizing controllers. Automatica, 38(11):1905-1917, 2002.

[6] C. Hoffmann and H. Werner. A survey of linear parametervarying control applications validated by experiments or high-fidelity simulations. IEEE Transactions on Control Systems Technology, 23(2):416-433, March 2015.

[7] I. Landau. On the use of Youla-Kucera parametrization in adaptive active noise and vibration control-A review. International Journal of Control, pages 1-25, 112018.

[8] B. Lu and F. Wu. Switching lpv control designs using multiple parameter-dependent lyapunov functions. Automatica, 40:1973-1980, 112004.

[9] I. Mahtout, F. Navas, D. Gonzalez, V. Milanes, and F. Nashashibi. Youla-kucera based lateral controller for autonomous vehicle. In 2018 21st International Conference on Intelligent Transportation Systems (ITSC), pages 32813286, Nov 2018.

[10] I. Mahtout, F. Navas, V. Milanés, and F. Nashashibi. Advances in Youla-Kucera parametrization: A Review. Annual Reviews in Control, June 2020. 
[11] F. Navas, V. Milanés, C. Flores, and F. Nashashibi. Multi-model adaptive control for cacc applications. IEEE Transactions on Intelligent Transportation Systems, pages $1-11,2020$

[12] J. Stoustrup. Plug\&play control: Control technology towards new challenges. European Journal of Control, pages 311-330, 2009.

[13] J. Stoustrup and H. Niemann. Starting up unstable multivariable controllers safely. In Proceedings of the 36th IEEE Conference on Decision and Control, volume 2, pages
1437-1438 vol.2, 1997.

[14] T. Tay, J. Moore, and I. Mareels. High performance control. Springer Science \& Business Media, 1997.

[15] M. Vidyasagar. Control system synthesis: a factorization approach, part ii. Synthesis lectures on control and mechatronics, 2(1):1-227, 2011.

[16] W. Xie and T. Eisaka. Design of lpv control systems based on youla parameterisation. IEE Proceedings - Control Theory and Applications, 151(4):465-472, July 2004. 